\title{
Erratum to: Mobility Management Strategies in Heterogeneous Cognitive Radio Networks
}

\section{Zoran Damljanović}

Published online: 30 January 2010

(C) Springer Science+Business Media, LLC 2010

\section{Erratum to: J Netw Syst Manage DOI 10.1007/s10922-009-9146-0}

In the original version of this paper 'Balasubramanyam $G$ ' reference and its citation is missing and it is cited below.

Under Section 4: Spectrum Selection (paragraph 2)

In a spectrum environment with multiple access technologies, different access networks have diverse characteristics and each access technology is suited for certain kinds of applications [32]. Thus, the CR's application has the possibility to use the most suitable access technology available [32].

Under Section 6.5: Cognitive Radio Session Layer (paragraph 2)

Upon CRSL receives information from the Cognitive Radio Application Layer to establish a connection to a new attachment point, the CRSL establishes a virtual connection to an attachment point $[31,32]$. The CRSL, as described in [32] "creates a new transport layer connection each time an end point moves to a new attachment point and manages the mapping of these transport layer connections to a virtual connection" [31].

\section{Reference}

32. Balasubramanyam, G.: Middleware architecture and protocols for multi-access aware mobile terminals. MSc thesis, Tampere University of Technology (2005)

The online version of the original article can be found under doi:10.1007/s10922-009-9146-0. 